\title{
Tissue factor is involved in retinoblastoma cell proliferation via both the Akt and extracellular signal-regulated kinase pathways
}

\author{
BYUNG JOO LEE ${ }^{1 *}$, JEONG HUN KIM ${ }^{1,2^{*}}$, SEUNG HOON WOO ${ }^{3}$, \\ JIN HYOUNG KIM ${ }^{1}$, DONG HUN KIM ${ }^{4}$ and YOUNG SUK YU ${ }^{1,2}$
}

\author{
${ }^{1}$ Fight against Angiogenesis-Related Blindness (FARB) Laboratory, Clinical Research Institute, Seoul National \\ University Hospital; ${ }^{2}$ Department of Ophthalmology, Seoul National University College of Medicine, Seoul; \\ ${ }^{3}$ Department of Otolaryngology and Institute of Health Sciences, College of Medicine, Gyeongsang National University, \\ Jinju; ${ }^{4}$ Department of Radiology, College of Medicine, Sooncheonhyang University, Bucheon, Republic of Korea
}

Received March 24, 2011; Accepted April 19, 2011

DOI: 10.3892/or.2011.1314

\begin{abstract}
Tissue factor (TF) is known to play a role in tumor progression. In retinoblastoma, the expression and role of TF has not been determined yet. Herein, we demonstrated for the first time that TF is closely related to the proliferation of retinoblastoma cells, which could be therefore effectively suppressed by blockade of the TF pathway. TF was selectively expressed on the areas of highly mitogenic activity in an orthotopic transplantation mouse model of retinoblastoma. In addition, the levels of TF expression in retinoblastoma cells were elevated after FGF2 treatment, whereas the proliferative effect of FGF2 on retinoblastoma cells was significantly inhibited by blockade of the TF pathway via TF pathway inhibitor (TFPI). Interestingly, retinoblastoma cells cultured with FGF2 showed increased phosphorylation of both Akt and ERK1/2. Addition of TFPI nearly abolished the FGF2induced phosphorylation of Akt and ERK1/2 in retinoblastoma cells. Therefore, our data suggest that TF expression in retinoblastoma cells is closely related to tumor cell proliferation and TFPI has the potential to inhibit retinoblastoma cell proliferation via the inhibition of both Akt and ERK1/2 activation.
\end{abstract}

\section{Introduction}

Retinoblastoma is the most common intraocular tumor in childhood which is hypervascular in nature and increased

Correspondence to: Dr Young Suk Yu, Department of Ophthalmology, College of Medicine, Seoul National University and Seoul Artificial Eye Center Clinical Research Institute, Seoul National University Hospital, Seoul 151-744, Republic of Korea

E-mail: ysyu@snu.ac.kr

${ }^{*}$ Contributed equally

Key words: Akt, extracellular signal-regulated kinase, proliferation, retinoblastoma, tissue factor, tissue factor pathway inhibitor vascular density of retinoblastoma is related to poor prognosis (1). Chong and colleagues found that extensive necrosis in retinoblastoma tissue indicates a poor prognosis and suggested thrombosis as a possible cause of extensive necrosis (2). Activation of coagulation is a common feature of several cancers and TF is a key molecule in cancer associated thrombosis $(3,4)$.

Tissue factor (TF), a transmembrane glycoprotein well documented as the initiator of coagulation pathway, is also involved in intracellular signaling independent of the coagulation pathway (5). TF forms a complex with coagulation factor VIIa and this complex acts through protease-activated receptors (PARs) to induce intracellular signals. TF is involved in diverse patho-physiological processes including angiogenesis and artherosclerosis via stimulating the proliferation of endothelial cell and vascular smooth muscle cells. In several cancer types, the expression of TF by tumor cells is related to tumor progression (6-8). It has been established that TF participates in tumor cell survival, tumor growth, angiogenesis and metastasis (6-10). TF is also known to promote mitosis of tumor cells (11).

In smooth muscle cells and human BOSC23 cells, ERK1/2 pathway was identified to mediate TF stimulated cell proliferation $(12,13)$, but still little is known about the mechanism responsible for TF-induced cellular proliferation, especially in tumor cells. TF could exert a role in promoting cell proliferation largely in two mechanisms. First, TF affects tumor cell proliferation through the activation of coagulation cascade. Thrombin is a mitogenic agent for fibroblast, endothelial cells and smooth muscle cells (14-16). In the presence of TF, thrombin mediated proliferation was identified in glioma cells (11). On the other hand TF could also affect tumor proliferation via TF-FVIIa-PAR signaling. Selective blocking of either coagulation or direct signaling function of TF showed that direct signaling through TF-FVIIa-PAR2 is sufficient for inhibiting the growth of breast cancer in a xenoplantation model (17).

Tissue factor pathway inhibitor (TFPI) is a natural trivalent Kunitz-type inhibitor of TF, which is mainly synthesized in the vascular endothelium. Domain 1 and 2 of TFPI inhibits 
the TF;VIIa complex (18) and domain 2 inactivates the TF:VIIa;Xa complex. Also, the function of domain 3 (K3) is still unclear, but recently, Hembrough et al reported that the anti-proliferative effect of TFPI relies on K3 and carboxylterminal at least in part and this action is mediated by VLDL receptor (19). TFPI is known to inhibit the proliferation of vascular endothelial cells and human neonatal aortic smooth muscle cells (19-21). However, there is scarce data on the exact intra-cellular signaling responsible for the TFPI induced inhibition of mitosis.

The expression of TF in retinoblastoma is undetermined and its possible role in retinoblastoma is unknown. In the present study, we tested the hypothesis that retinoblastoma cells express $\mathrm{TF}$, the expression of $\mathrm{TF}$ is related to the proliferation of retinoblastoma cells and whether TFPI inhibits the proliferation of retinoblastoma cells. Finally, we evaluated the downstream signal pathways responsible for $\mathrm{TF}$ associated retinoblastoma cell proliferation.

\section{Materials and methods}

Cell culture. Y-79 (22) human retinoblastoma cell line which was purchased from American Type Culture Collection (Manassas, VA, USA) and SNUOT-Rb1 (23), a spontaneously immortalized human retinoblastoma cell line established by our group were used at passage 3-7. These cells were cultured at $37^{\circ} \mathrm{C}$ in RPMI-1640 (WelGene, South Korea) containing penicillin-streptomycin $(10 \mathrm{ml} / \mathrm{l}$, Gibco-Invitrogen Corporation, Paisley, UK) with $10 \%$ fetal bovine serum (Gibco-Invitrogen Corporation). The culture media were refreshed every 3 days. For the induction of mitogenic stimuli, $18 \mathrm{kDa}$ exogenous FGF-2 was used. FGF-2 of 18-, 22-, and 34-kDa isoforms are known to be expressed in the retinoblastoma cell lines and similar isoforms are found in LHBETATAG transgenic mouse $(24,25)$. Cebulla et al reported that the $18 \mathrm{kDa}$ exogenous FGF-2 induces the proliferation of WERI and Y-79 cell lines (24). Sub-confluent cells were treated with or without $18 \mathrm{kDa}$ recombinant FGF-2 $(5 \mathrm{ng} / \mathrm{ml})$ as a mitogen in combination with full-length recombinant human TFPI of various concentrations $(0-100 \mathrm{ng} / \mathrm{ml})$ for $24 \mathrm{~h}$. Since Hembrough et al found that the antiproliferative activity of TFPI is diminished when Kunitz-3 and the carboxyl-terminus of TFPI is truncated (19), we used a full-length recombinant human TFPI which was purchased from American diagnostica.

Orthotopic transplantation mouse model of retinoblastoma. For inducing intraocular tumorigenesis, Y79 cells were injected into the intravitreal cavity of BALB/c-nude mice as our group previously described (23). Cultivated Y79 cells were harvested, and suspended in cold PBS $\left(4^{\circ} \mathrm{C}\right)$, a concentration of $2.5 \times 10^{6}$ cells per $20 \mu \mathrm{l}$, and then injected into intravitreal cavity of mice using 30-gauge needle. Four weeks after injection, mice were examined for adequate tumorigenesis, and enucleated.

Histological examination and immunohistochemistry. Formalin-fixed, paraffin embedded blocks of tumors were sectioned $(4 \mu \mathrm{m})$ and were de-paraffinized through xylene and alcohols. After the de-paraffinization, tumor sections were incubated with proteinase $\mathrm{K}$ at $37^{\circ} \mathrm{C}$. Then treated with $3 \%$
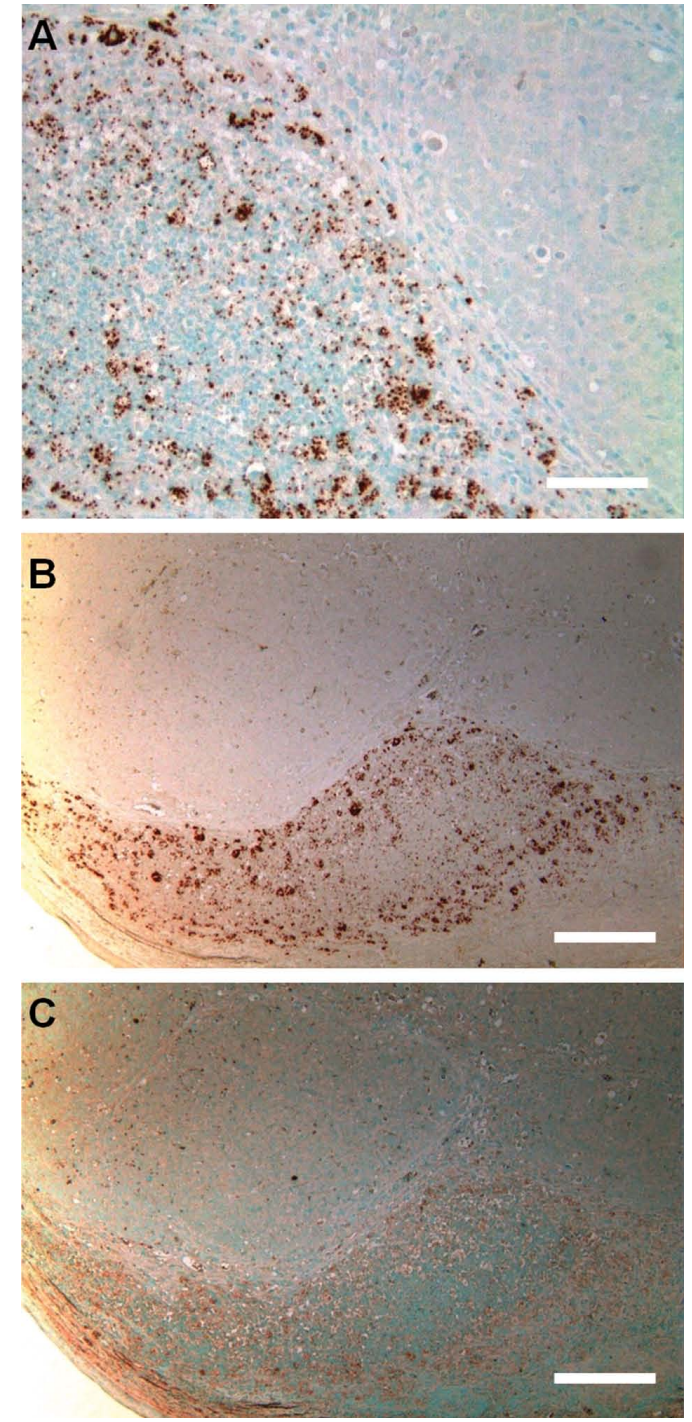

Figure 1. TF is selectively expressed by a sub-population of retinoblastoma cells with highly mitogenic activity in xenoplantation mouse model of retinoblastoma. Four weeks after the intravitreal injection of Y-79 cells, tumor filled the vitreal cavity of BALB/c-nude mice. TF immunoreactivity is prominent in the area of retinoblastoma cells with high nuclear/cytosolic ratio while the area with low nuclear/cytosolic ratio is not stained with anti-TF antibody. Scale bar: $50 \mu \mathrm{m}$ (A). In a serial section, TF expressing tumor cells (B) showed strong immunoreactivity to Ki-67 whereas the adjacent TF negative areas showed relatively weak immunoreactivity (C). Scale bars: $200 \mu \mathrm{m}$.

hydrogen peroxide to block endogenous peroxidase activity and with blocking kit (Zymed Laboratories, San Francisco, CA, USA) to avoid nonspecific binding. Prepared sections were incubated overnight with 1:100 anti-TF rabbit polyclonal antibody (sc-30201, Santa Cruz Biotechnology, CA, USA) at $4^{\circ} \mathrm{C}$. After rinsing with PBS, sections were incubated with goat anti-rabbit biotinylated secondary antibody (Invitrogen) and followed by streptavidin horseradish peroxidase. The bound antiserum was visualized by incubating with 3-amino9-ethylcabazole (AEC, Dako Company, Carpinteria, CA, USA). After counterstained with Methyl green, sections were observed by light microscopy. Same procedure was performed with 1:200 anti-Ki-67 rabbit polyclonal antibody (ab15580, Abcam, Cambridge, UK). Primary antibodies were omitted for negative control. 
3-(4,5-Dimethylthiazol-2-yl)-2,5-diphenyltetrazolium bromide (MTT) assay. The degree of cellular proliferation was quantified by estimating the number of viable cells with the MTT assay. Cells (1x10 $/$ well) were plated in 96-well plates and cultured overnight. Cells were cultured with FGF-2 (5 ng/ml, Chemicon International), TFPI (American Diagnostica) and signal pathway inhibitors of various concentrations $(0,10,100 \mathrm{ng} / \mathrm{ml})$ for $24 \mathrm{~h}$. The medium was then replaced with the mixture of fresh culture medium and $0.5 \mathrm{mg} / \mathrm{ml}$ MTT solution. Cells were incubated for $2 \mathrm{~h}$ at $37^{\circ} \mathrm{C}, 5 \% \mathrm{CO}_{2}$ and then, re-incubated for 10 min with DMSO at $37^{\circ} \mathrm{C}$. Following the reaction, absorbance was measured at $540 \mathrm{~nm}$. The absorbance from treatment groups was compared with untreated controls, cultured with standard serum containing medium.

Western blot analysis. The expression of TF and the phosphorylation status of Akt and ERK1/2 were determined by Western blot analysis. Cells were treated according to the previously mentioned protocol. After the treatment, cells were harvested and lysed for protein extraction. The protein concentration in the cytosolic fraction was measured using a BCA protein assay kit (Pierce, Rockford, IL, USA). Protein extracts ( $20 \mu \mathrm{g}$ for each) were separated by gel electrophoresis and then transferred to nitrocellulose membranes. Membranes were blocked for $30 \mathrm{~min}$ with skimmed milk for the prevention of nonspecific binding and incubated overnight at $4^{\circ} \mathrm{C}$ with primary antibodies (anti-TF, anti-Akt, anti phospho-Akt, anti-ERK1/2, anti phospho-ERK1/2, and anti- $\beta$-actin antibodies) in the concentration of 1:1000. Washed membranes were re-incubated with secondary antibodies (horseradish peroxidaseconjugated anti-rabbit or anti-mouse $\mathrm{IgG}$ ) of 1:5000 dilution, and detected by ECL Plus ${ }^{\mathrm{TM}}$ Western Blotting Reagent Pack (GE Healthcare, Piscataway, USA). The blots were scanned using a flatbed scanner and the band intensity analysed using the TINA software program (Raytest, Staubenhardt, Germany).

Statistical analysis. Statistical differences between groups were evaluated with the Mann-Whitney U test. The cut-off value of statistical significance was set at $\mathrm{p}<0.05$.

\section{Results}

Selective expression of TF in xenoplantation mouse model of retinoblastoma. Four weeks after the inoculation of Y-79 cell line, vitreal cavity was nearly completely occupied by tumor. In the xenograft mouse model of retinoblastoma, TF and Ki-67 expression was determined by immunohistochemical analysis. Only some tumor cells were recognized by TF antibodies. TF immunopositive cells showed high nuclear/ cytosolic ratio than adjacent TF immunonegative cells (Fig. 1A). In a serial section, TF immunopositive cells (Fig. 1B) demonstrated stronger Ki-67 positivity (Fig. 1C) than TF immunonegative cells. This result could be interpreted that TF is selectively expressed by tumor cells with a high mitogenic activity.

The expression and FGF-2 induced up-regulation of TF in human retinoblastoma cell line. To determine whether TF is expressed by human retinoblastoma cells and whether mitogenic stimuli changes the level of TF expression in human

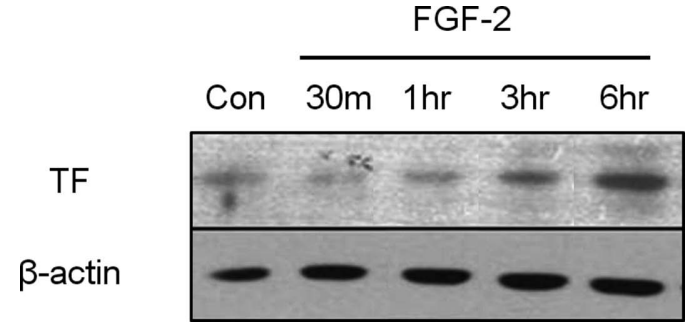

Figure 2. Time-dependent effects of FGF-2 on the expression of TF in the SNUOT-Rb1 cells. SNUOT-Rb1 cells cultured under standard medium supplemented with $10 \%$ fetal bovine serum showed basal expression of TF. When treated with FGF-2 $(5 \mathrm{ng} / \mathrm{ml})$, level of TF expression in SNUOT-Rb1 cells was increased along the time interval. The level of TF reached a peak $6 \mathrm{~h}$ after treatment.

retinoblastoma cells, SNUOT-Rb1 cells were treated with FGF-2 (5 ng/ml). FGF-2 is a well-known strong mitogen which is reported to be produced in human retinoblastoma (25). Exogenous FGF-2 is also reported to induce the proliferation of human retinoblastoma cell lines (Y-79 and WERI) in a dose-dependent manner (24). SNUOT-Rb1 cells showed a basal expression of TF under standard medium. When cells were exposed to FGF-2, the total protein level of TF was significantly increased from the first hour and sustained till the 6th hour (Fig. 2).

The reversal of FGF-2-induced proliferation of human retinoblastoma cell by TFPI. The effect of TFPI on the FGF-2 stimulated proliferation of retinoblastoma cell was assessed by MTT assay. First, the level of cellular proliferation in response to exogenous FGF-2 $(5 \mathrm{ng} / \mathrm{ml})$ was evaluated with MTT assay. As described previously, same number of SNUOT- Rb1 cells ( $1 \times 10^{5}$ cells/well) were plated in a 96-well plate and treated with FGF-2 $(5 \mathrm{ng} / \mathrm{ml})$ under various concentration of TFPI $(0,10,100 \mathrm{ng} / \mathrm{ml})$. After $24 \mathrm{~h}$, SNUOT-Rb1 cells treated with exogenous FGF-2 showed increased level of relative density $(1.42 \pm 0.18)$ compared to untreated controls $(\mathrm{p}=0.020)$, in concordance with a previous study (24) When SNUOT-Rb1 cells were co-treated with FGF-2 and TFPI of a concentration of $10 \mathrm{ng} / \mathrm{ml}$ showed decreased density $(1.10 \pm 0.19)$ compared to cells treated with FGF-2 alone, but there was no statistical significance $(p=0.070)$. The anti-proliferative effect of TFPI against FGF-2 became significant $(\mathrm{p}=0.002)$ at a concentration of $100 \mathrm{ng} / \mathrm{ml}$. With this concentration of TFPI, the relative density $(1.04 \pm 0.13)$ did not showed significant difference $(p=0.294)$ from that of control (Fig. 3). On the other hand, SNUOT-Rb1 cells treated only with TFPI did not show significant decrement of density compared to untreated controls (data not shown).

FGF-2 induces activation of both Akt and ERK1/2. As the ERK1/2 and Akt activation are well known intracellular signals involved in the cellular proliferation, we evaluated the change in activities of these signals after FGF-2 treatment in retinoblastoma cells. Following FGF-2 treatment, the phosphorylation of Akt was immediately increased at $10 \mathrm{~min}$ and then decreased to the level of control within 30 min after the treatment (Fig. 4). FGF-2 also induced the phosphorylation of ERK1/2 which was gradually increased in a time-dependent 


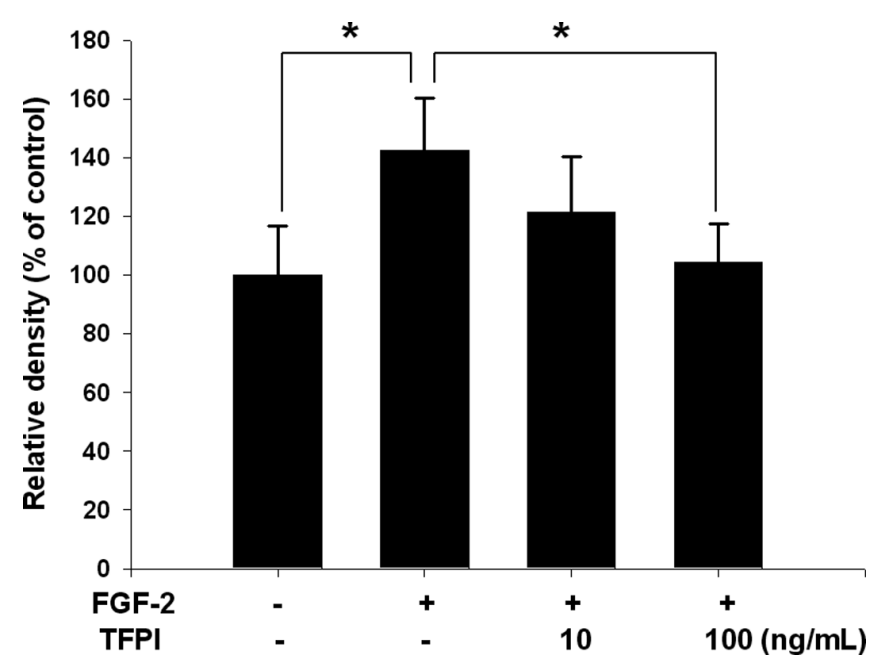

Figure 3. TFPI inhibits FGF-2 induced proliferation of SNUOT-Rb1. SNUOT Rb-1 cells were cultivated with or without FGF-2 $(5 \mathrm{ng} / \mathrm{ml})$ in addition to various concentrations of TPFI (10 and $100 \mathrm{ng} / \mathrm{ml})$. Cellular population was determined with MTT assay $24 \mathrm{~h}$ after treatment. FGF-2 treated group showed a 1.42-fold increment of cellular population compared to control $(\mathrm{p}=0.020)$. Co-treatment with TFPI $100 \mathrm{ng} / \mathrm{ml}$ suppressed the proliferative effect of FGF-2 ( $\mathrm{p}=0.002)$ and the relative density of this group did not show statistically significant difference from that of control $(p=0.249)$. Data represent the means \pm SE from three independent experiments $\left({ }^{*} \mathrm{p}<0.05\right)$

manner and reach to the peak level at $3 \mathrm{~h}$, then decreased to pre-treatment level (Fig. 4).

TFPI inhibits FGF-2-induced activation of both ERK1/2 and $A K t$. Since TFPI effectively inhibits FGF-2 induced proliferation of retinoblastoma cells, we decided to assess the effect of TFPI on the intracellular signals which are evoked by proliferative stimuli of FGF-2. Retinoblastoma cells were incubated with $5 \mathrm{ng} / \mathrm{ml} \mathrm{FGF-2} \mathrm{and} 100 \mathrm{ng} / \mathrm{ml}$ TFPI the concentration of which was found to inhibit FGF-2 stimulated proliferation of retinoblastoma cells by MTT assay. After 10 min, cells co-treated with FGF-2 and TPFI showed significantly decreased level of Akt phosphorylation compared to controls treated with FGF-2 alone (Fig. 4). TFPI also affected the phosphorylation status of ERK1/2 from the 10th minute and this suppressive effect on ERK1/2 phosporylation was sustained thereafter (Fig. 4).

\section{Discussion}

Previous clinicopathologic studies have revealed that TF is expressed in tumors and the level of TF expression is different according to degree of histologic grading $(26,27)$. This is the first study to our knowledge that TF is expressed in retinoblastoma cells. Our study showed a selective expression of TF in xenoplantation mouse model of retinoblastoma. According to our result, TF is not expressed in all retinoblastoma cells, but only expressed in the cells with strong mitotic activity. We also found a concentration-dependent up-regulation of TF on retinoblastoma cells after FGF-2 treatment. Still little is known about the effect of FGF on the expression of TF except for endothelial cells. In HUVEC, FGF has been reported to suppress the transcription of TF (28). Therefore, our result of FGF2 induced TF up-regulation in retinoblastoma cell is a novel finding which is contrary to previous results. Above findings show a bidirectional correlation of TF and proliferative stimulus in the retinoblastoma cells.

The mechanism by which TF is involved in tumor growth is not clear yet, but several studies implicated that direct signaling by TF-VIIa complex independent of coagulation cascade is important in tumor progression. A recent study using monoclonal antibody which specifically blocks TF-VIIa signaling revealed that the TF-VIIa complex induces tumor growth via activation of PAR2 (17). However, the downstream pathways responsible for mitogenic stimuli are not fully understood. Some authors postulated that the activation of ERK1/2 is indispensible for PAR mediated cellular proliferation $(12,29)$.

In general, mitogen activated protein kinase (MAPK) pathways and PI3K/Akt pathways are two major intracellular signal pathways involved in cellular proliferation. The role of PI3K/Akt is well documented in endothelial cell proliferation. Sulpice et al showed that the activation of both ERK1/2 and PI3K/Akt is required for the FGF-2 induced proliferation of adrenal cortex capillary endothelial cells (30). In malignant cells, the activation of ERK1/2 has been reported to be important in FGF-2 stimulated synovial sarcoma proliferation (31). In this study, exogenous $18 \mathrm{kDa}$ recombinant FGF-2 induced proliferation of SNUOT-Rb1 cells and activated both PI3K/Akt and ERK1/2 pathways in the concentration which resulted in cellular proliferation.

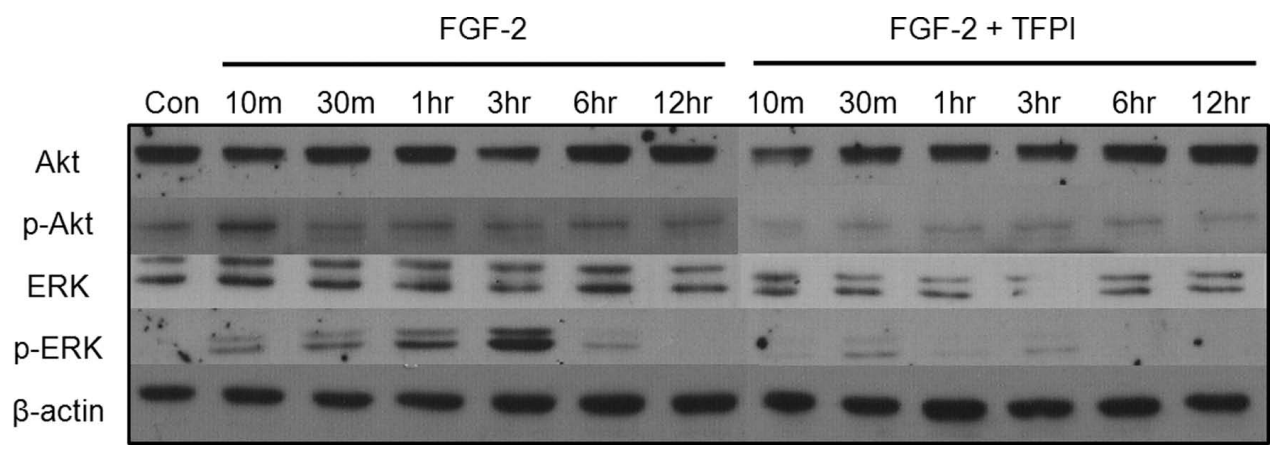

Figure 4. TFPI inhibits FGF-2 induced activation of both ERK1/2 and PI3K/AKt. SNUOT-Rb1 cells were treated with FGF-2 (5 ng/ml) with and without TFPI $(100 \mathrm{ng} / \mathrm{ml})$ and incubated for $12 \mathrm{~h}$. At each time point, the phosphorylation status of Akt and ERK1/2 was evaluated by Western blot anlaysis. In the FGF-2 treated group, increased phosphorylation of both Akt and ERK1/2 was noted at different time points, whereas these effects were abolished in the group co-treated with TFPI (100 ng/ml). 
The anti-proliferative effect of TFPI has been reported in endothelial cells and vascular smooth muscle cells. However, the mechanism of anti-proliferative effect of TFPI has to be elucidated. FGF-2-induced proliferation could be affected by TFPI in several mechanisms. First, TFPI could alter FGF-2 itself. Yu et al found that thrombin induces cellular proliferation by cleaving anti-proliferative high molecular weight (HMW) FGF-2 into pro-proliferative low molecular weight (LMW) FGF-2 (32). In this study, to exclude the possibility that FGF-2 cleavage itself is affected by TFPI-induced suppression of prothrombin conversion into thrombin, we used LMW FGF-2 which does not need cleavage.

The inhibitory effect of TFPI on the retinoblastoma cell proliferation could be either dependent or independent of coagulation pathway. TF-VIIa-dependent PAR2-mediated signal or TF-independent different pathway could be the coagulation pathway independently on which TFPI exerts anti-proliferative activity. Hembrough et al suggested that the anti-proliferative effect might not depend on the inhibition of TF-VIIa induced signaling, but partially through the VLDL receptor in endothelial cells (19). However, specific polyclonal antibody to the VLDL receptor could not fully abolished TFPI-induced inhibition of endothelial cell proliferation. This implies another mechanism is required for understanding the anti-proliferative effect of TFPI. In this aspect, the antiproliferative effect of TFPI on retinoblastoma cells could be at least partially explained by the inhibitory activity of TFPI on TF-Vlla complex induced signaling. Moreover, the fact that both PI3K/Akt and ERK1/2 which are representative signal downstream to PAR were inactivated by TFPI may suggest a possible role of PAR in the suppressive effect of TFPI on FGF2 induced proliferation.

SNUOT-Rb1 cultured with TFPI and FGF-2 showed decreased proliferation compared with the group which was treated with FGF-2 alone, reaching nearly the level of untreated control group. However, TFPI alone did not demonstrate significant inhibitory effect on SNUOT-Rb1 cell proliferation. There could be three possible explanations for this phenomenon. First, Rauch and co-workers previously found that FGF2 is required for the thrombin and FXa-induced, PAR-1 mediated DNA synthesis (33). Our finding that the antagonistic effect of TFPI against retinoblastoma cell proliferation only worked under the supplement of exogenous FGF2 might be explained by this permissive role of FGF2. Second, it is known that FGF2 selectively up-regulates PAR2 which is important in TF induced mitogenic activity (34). FGF2 may also up-regulate PAR-2 in retinoblastoma cells and make them sensitive to the suppressive effect of FGF2-induced proliferation by TFPI. Third, FGF-2 mediated overexpression of TF might have influenced the inhibitory effect of the TFPI on SNUOT-Rb1 cells. In quiescent status, the retinoblastoma cell itself expresses low level of TF, but on proliferative stimulus, the expression of TF is increased, and then TFPI may exert antiproliferative effect via inactivation of the TF-Vlla complex.

Collectively, TF is selectively expressed in retinoblastoma cells, and resulting in tumor cell proliferation and survival. TF induced retinoblastoma proliferation depends on the activation of signals induced by FVIIa-TF complex and both Akt and ERK1/2 pathways are associated with FGF-2 induced retinoblastoma cell proliferation. TFPI is a potential candidate for human retinoblastoma treatment, but further study using an animal model is required.

\section{Acknowledgements}

This study was supported by a grant from the National R\&D program for cancer control, Ministry of Health and Welfare, Republic of Korea (800-20100332) and by a grant from Seoul National University Hospital (0420100590).

\section{Reference}

1. Marback EF, Arias VE, Paranhos A Jr, Soares FA, Murphree AL and Erwenne CM: Tumour angiogenesis as a prognostic factor for disease dissemination in retinoblastoma. Br J Ophthalmol 87: 1224-1228, 2003.

2. Chong EM, Coffee RE, Chintagumpala M, Hurwitz RL, Hurwitz MY and Chevez-Barrios P: Extensively necrotic retinoblastoma is associated with high-risk prognostic factors. Arch Pathol Lab Med 130: 1669-1672, 2006.

3. Bick RL: Cancer-associated thrombosis. N Engl J Med 349: 109-111, 2003.

4. Lee AY: Thrombosis and cancer: the role of screening for occult cancer and recognizing the underlying biological mechanisms. Hematology Am Soc Hematol Educ Program 2006: 438-443, 2006.

5. Bromberg ME, Konigsberg WH, Madison JF, Pawashe A and Garen A: Tissue factor promotes melanoma metastasis by a pathway independent of blood coagulation. Proc Natl Acad Sci USA 92: 8205-8209, 1995.

6. Contrino J, Hair G, Kreutzer DL and Rickles FR: In situ detection of tissue factor in vascular endothelial cells: correlation with the malignant phenotype of human breast disease. Nat Med 2: 209-215, 1996.

7. Seto S, Onodera H, Kaido T, et al: Tissue factor expression in human colorectal carcinoma: correlation with hepatic metastasis and impact on prognosis. Cancer 88: 295-301, 2000.

8. Khorana AA, Ahrendt SA, Ryan CK, et al: Tissue factor expression, angiogenesis, and thrombosis in pancreatic cancer. Clin Cancer Res 13: 2870-2875, 2007.

9. Abdulkadir SA, Carvalhal GF, Kaleem Z, et al: Tissue factor expression and angiogenesis in human prostate carcinoma. Hum Pathol 31: 443-447, 2000.

10. Poon RT, Lau CP, Ho JW, Yu WC, Fan ST and Wong J: Tissue factor expression correlates with tumor angiogenesis and invasiveness in human hepatocellular carcinoma. Clin Cancer Res 9: 5339-5345, 2003.

11. Ogiichi T, Hirashima Y, Nakamura S, Endo S, Kurimoto M and Takaku A: Tissue factor and cancer procoagulant expressed by glioma cells participate in their thrombin-mediated proliferation. J Neurooncol 46: 1-9, 2000.

12. Cirillo P, Cali G, Golino P, et al: Tissue factor binding of activated factor VII triggers smooth muscle cell proliferation via extracellular signal-regulated kinase activation. Circulation 109: 2911-2916, 2004.

13. Fan L, Yotov WV, Zhu T, et al: Tissue factor enhances proteaseactivated receptor-2-mediated factor VIIa cell proliferative properties. J Thromb Haemost 3: 1056-1063, 2005.

14. Gospodarowicz D, Brown KD, Birdwell CR and Zetter BR: Control of proliferation of human vascular endothelial cells. Characterization of the response of human umbilical vein endothelial cells to fibroblast growth factor, epidermal growth factor, and thrombin. J Cell Biol 77: 774-788, 1978.

15. Carney DH, Stiernberg J and Fenton JW II: Initiation of proliferative events by human alpha-thrombin requires both receptor binding and enzymic activity. J Cell Biochem 26: 181-195, 1984.

16. Chen LB and Buchanan JM: Mitogenic activity of blood components. I. Thrombin and prothrombin. Proc Natl Acad Sci USA 72: 131-135, 1975.

17. Versteeg HH, Schaffner F, Kerver M, et al: Inhibition of tissue factor signaling suppresses tumor growth. Blood 111: 190-199, 2008.

18. Girard TJ, Warren LA, Novotny WF, et al: Functional significance of the Kunitz-type inhibitory domains of lipoproteinassociated coagulation inhibitor. Nature 338: 518-520, 1989. 
19. Hembrough TA, Ruiz JF, Papathanassiu AE, Green SJ and Strickland DK: T inhibits endo-thelial cell proliferation via association with the very low density lipoprotein receptor. J Biol Chem 276: 12241-12248, 2001.

20. Hembrough TA, Ruiz JF, Swerdlow BM, et al: Identification and characterization of a very low density lipoprotein receptor-binding peptide from tissue factor pathway inhibitor that has antitumor and antiangiogenic activity. Blood 103: 3374-3380, 2004.

21. Kamikubo Y, Nakahara Y, Takemoto S, Hamuro T, Miyamoto S and Funatsu A: Human recombinant tissue-factor pathway inhibitor prevents the proliferation of cultured human neonatal aortic smooth muscle cells. FEBS Lett 407: 116-120, 1997.

22. Reid TW, Albert DM, Rabson AS, et al: Characteristics of an established cell line of retinoblastoma. J Natl Cancer Inst 53 347-360, 1974

23. Kim JH, Yu YS, Kim DH, Kim CJ and Kim KW: Establishment and characterization of a novel, spontaneously immortalized retinoblastoma cell line with adherent growth. Int J Oncol 31: 585-592, 2007

24. Cebulla CM, Jockovich ME, Pina Y, et al: Basic fibroblast growth factor impact on retinoblastoma progression and survival. Invest Ophthalmol Vis Sci 49: 5215-5221, 2008.

25. Schweigerer L, Neufeld G and Gospodarowicz D: Basic fibroblast growth factor is present in cultured human retinoblastoma cells. Invest Ophthalmol Vis Sci 28: 1838-1843, 1987.

26. Nitori N, Ino Y, Nakanishi Y, et al: Prognostic significance of tissue factor in pancreatic ductal adenocarcinoma. Clin Cancer Res 11: 2531-2539, 2005.

27. Hamada K, Kuratsu J, Saitoh Y, Takeshima H, Nishi T and Ushio Y: Expression of tissue factor correlates with grade of malignancy in human glioma. Cancer 77: 1877-1883, 1996.
28. Pendurthi UR, Williams JT and Rao LV: Acidic and basic fibroblast growth factors suppress transcriptional activation of tissue factor and other inflammatory genes in endothelial cells. Arterioscler Thromb Vasc Biol 17: 940-946, 1997.

29. Darmoul D, Gratio V, Devaud H and Laburthe M: Proteaseactivated receptor 2 in colon cancer: trypsin-induced MAPK phosphorylation and cell proliferation are mediated by epidermal growth factor receptor transactivation. J Biol Chem 279 : 20927-20934, 2004.

30. Sulpice E, Bryckaert M, Lacour J, Contreres JO and Tobelem G: Platelet factor 4 inhibits FGF2-induced endothelial cell proliferation via the extracellular signal-regulated kinase pathway but not by the phosphatidylinositol 3-kinase pathway. Blood 100: 3087-3094, 2002.

31. Ishibe T, Nakayama T, Okamoto T, et al: Disruption of fibroblast growth factor signal pathway inhibits the growth of synovial sarcomas: potential application of signal inhibitors to molecular target therapy. Clin Cancer Res 11: 2702-2712, 2005.

32. Kim JH, Yu YS, Kim DH, Kim YK and Kim KW: Comparative genomic hybridization analysis of newly established retinoblastoma cell lines of adherent growth compared with Y79 of nonadherent growth. J Pediatr Hematol Oncol 30: 571-574, 2008.

33. Rauch BH, Millette E, Kenagy RD, Daum G and Clowes AW: Thrombin- and factor Xa-induced DNA synthesis is mediated by transactivation of fibroblast growth factor receptor-1 in human vascular smooth muscle cells. Circ Res 94: 340-345, 2004.

34. Abe K, Aslam A, Walls AF, Sato T and Inoue H: Up-regulation of protease-activated receptor- 2 by bFGF in cultured human synovial fibroblasts. Life Sci 79: 898-904, 2006. 